\title{
Inotropic Responses to Selective (RO 20-1724 and SQ 65,442) and Nonselective (Trequinsin) Inhibitors of Cyclic AMP-Specific Class IV Phosphodiesterase in Newborn, Immature, and Adult Rabbit Myocardium
}

\author{
NIKI L. OQUIST, SAMUEL J. STRADA, AND MICHAEL ARTMAN \\ Departments of Pediatrics and Pharmacology, University of South Alabama College of Medicine, \\ Mobile, Alabama 36617
}

\begin{abstract}
In contrast to myocardium from adult rabbits, myocardium from newborns is insensitive to the inotropic effects of selective inhibitors (e.g. amrinone, milrinone, and indolidan) of the cGMP-inhibited high-affinity cAMP phosphodiesterase (PDE) localized in the sarcoplasmic reticulum. This difference may be explained at least partially by our recent observation that this cAMP PDE activity is low in sarcoplasmic reticulum from newborns. Furthermore, because the predominant cytosolic high-affinity cAMP PDE activity in newborns is a cGMPinsensitive form, we postulated that selective inhibitors of this form of cAMP-specific PDE may increase cardiac contractility in newborns. Therefore, the inotropic effects of RO 20-1724 and SQ 65,442 (selective inhibitors of cGMP-insensitive, high-affinity cAMP PDE) were compared with trequinsin (a potent, less selective PDE inhibitor) in right ventricular papillary muscles isolated from newborn (NB; 24-48 h), immature (14-16 d), and adult New Zealand White rabbits. At a drug concentration of $100 \mu \mathrm{m}$, RO 20-1724 and SQ 65,442 depressed maximal rate of tension development to $67 \pm 4$ and $70 \pm 2 \%$ of control, respectively, in NB papillary muscles. The NB response to RO $20-1724$ differed significantly from the immature $(127 \pm 2 \%)$ and adult $(115 \pm 3 \%)$ groups $(p<$ 0.05), but the effects of SQ 65,442 were comparable among the three age groups. In contrast, trequinsin exerted a positive inotropic effect in the NB group (355 $\pm 22 \%$ of control) that was substantially greater than the maximal response obtained in the immature $(139 \pm 6 \%$ of control) or adult $(131 \pm 5 \%$ of control) groups $(p<0.01)$. These differences in inotropic responsiveness could not be explained by differences in sensitivity to drug-induced inhibition of cAMP or cGMP hydrolysis in cytosolic fractions from the three age groups. Thus, selective inhibitors of the predominant form of high-affinity cAMP PDE in NB myocardium do not exert a positive inotropic response in NB papillary muscles. The age-specific cardiotonic effects of trequinsin suggest that other PDE isozymes could contribute to the modulation of contractility during postnatal maturation. (Pediatr Res 31: 300-304, 1992)
\end{abstract}

Received June 10, 1991; accepted October 16, 1991

Correspondence: Michael Artman, M.D., Department of Pediatrics, University of South Alabama Medical Center, 2451 Fillingim St., Mobile, AL 36617.

Supported in part by the American Heart Association Alabama Affiliate, Inc. Burroughs Wellcome New Investigator Award (M.A.), and the Pharmaceutical Manufacturers Association Foundation, Inc. M.A. is the recipient of a USPHS Clinical Investigator Award from the NHLBI (HL-01695)
Abbreviations

PDE, phosphodiesterase

SR, sarcoplasmic reticulum

$\mathrm{dT} / \mathrm{dt}$, maximal rate of tension development

NB, newborn

IM, immature

$\mathrm{AD}$, adult

$\mathrm{IC}_{50}$, concentration producing $\mathbf{5 0 \%}$ inhibition

$\mathrm{RT}$, resting tension

$-\mathrm{dT} / \mathrm{dt}$, maximal rate of relaxation

Cyclic nucleotide PDE play a central role in the modulation of contractile function in mature myocardium. Physiologic or pharmacologic interventions that increase intracellular cAMP (such as $\beta$-adrenergic stimulation) result in activation of cAMPdependent protein kinase, phosphorylation of key regulatory proteins, and increased contractility (1-5). Hydrolysis of cAMP is catalyzed by a family of cyclic nucleotide PDE. Thus, the distribution and activity of cAMP-specific PDE are important determinants of the inotropic state and drug responsiveness.

Cyclic nucleotide PDE can be classified according to their relative substrate affinities and effects of cGMP and $\mathrm{Ca}^{2+} / \mathrm{cal}-$ modulin on hydrolytic activity (6). High-affinity cAMP PDE activity exists in two major forms in mammalian myocardium: the class III cGMP-inhibited PDE that is also sensitive to inhibition by cardiotonic drugs such as milrinone and a cardiotonic drug-insensitive form that is not sensitive to inhibition by cGMP. This cGMP-insensitive form belongs to the class IV (cAMPspecific) PDE family. PDE activity in the SR is almost exclusively the CGMP-inhibited class III PDE (7-12). In contrast, the soluble or cytosolic fraction of rabbit myocardium contains both cGMPinhibited (class III) and cGMP-insensitive (class IV) high-affinity cAMP PDE activities $(7,12)$.

Evidence has accumulated to support the concept that the major site of action of cardiotonic PDE inhibitors such as milrinone, indolidan, and imazodan is the SR-associated cGMPinhibited class III PDE (7-13). Myocardium from NB animals is relatively insensitive to this class of agents (14-17), consistent with our observation of diminished cGMP-inhibited class III PDE activity in NB SR (12). Unlike that in AD rabbits, highaffinity cAMP PDE activity in the cytosolic fraction from NB myocardium is predominantly the class IV, cGMP-insensitive, cardiotonic drug-insensitive form (12). Therefore, we postulated that selective inhibitors of the predominant form of high-affinity cAMP PDE activity in NB myocardium (the cGMP-insensitive 
class IV form) would produce a positive inotropic effect in the NB heart.

This hypothesis was tested by determining the inotropic responses to two different selective inhibitors of cAMP-specific class IV PDE (RO 20-1724 and SQ 65,442) (7, 18-21) during postnatal maturation in the rabbit. Results were compared with those obtained using a potent, but more generalized PDE inhibitor (trequinsin) $(21,22)$. To relate the inotropic responses to inhibition of PDE activity, cytosolic fractions from each age group were assayed for sensitivity to inhibition of cAMP and cGMP hydrolysis.

\section{MATERIALS AND METHODS}

Animals. All experiments were performed using New Zealand White rabbits of either sex from three age groups: NB, 24 to 48 h of age; IM, 14 to $16 \mathrm{~d}$ of age; and sexually mature AD, 6 to 8 mo of age. Timed-pregnant rabbits delivered in our animal care facility to allow precise aging of the litters. NB and IM rabbits were housed with their mothers. Hearts from lactating rabbits were not used for these experiments. All animals were deeply anesthetized with sodium pentobarbital (50-75 mg/kg) administered intraperitoneally (NB and IM) or i.v. (AD) before removal of the heart. Our laboratory follows the highest standards of humane care and adheres to NIH guidelines for proper animal care. These experimental protocols were reviewed and approved by the University of South Alabama Institutional Animal Care and Use Committee.

Contractile responses. Each heart was rapidly excised and placed in ice-cold normal saline. Right ventricular papillary muscles were removed immediately and prepared to contract isometrically in Krebs bicarbonate buffer $(125 \mathrm{mM} \mathrm{NaCl}, 2.3$ $\mathrm{mM} \mathrm{KCl}, 2.5 \mathrm{mM} \mathrm{CaCl}_{2}, 25 \mathrm{mM} \mathrm{NaHCO}, 5.6 \mathrm{mM}$ dextrose, $1.3 \mathrm{mM} \mathrm{KH}_{2} \mathrm{PO}_{4}$ and $0.6 \mathrm{mM} \mathrm{MgSO}$ ) at $30^{\circ} \mathrm{C}$. A mixture of $95 \%$ oxygen and $5 \%$ carbon dioxide was continuously bubbled through the buffer ( $\mathrm{pH} 7.35-7.4)$. Each muscle was stimulated to contract using a square-wave pulse of $3 \mathrm{~ms}$ duration at a frequency of $0.5 \mathrm{~Hz}$. Muscles were gradually stretched to the optimum length for maximal tension development, then allowed to equilibrate for $20-30 \mathrm{~min}$ before drug exposure. Only muscles with a cross-sectional area $\leq 1 \mathrm{~mm}^{2}$ were included in the analyses. Cross-sectional area was calculated from the length and wet weight of each muscle at the completion of each experiment. The muscle length was measured from the calibrated micromanipulator used to adjust the height of the force transducer. Each muscle was removed from the clamp, the base and chordae were trimmed away, the muscle was blotted, and the wet weight was determined.

$\mathrm{RT}$, developed tension, time to peak tension, $\mathrm{dT} / \mathrm{dt}$, time from peak tension to $90 \%$ relaxation, and $-\mathrm{dT} / \mathrm{dt}$ were averaged for four consecutive contractions for any given conditions. Developed tension, $\mathrm{dT} / \mathrm{dt},-\mathrm{dT} / \mathrm{dt}$, and $\mathrm{RT}$ were normalized to 1 $\mathrm{mm}^{2}$ cross-sectional area to facilitate comparisons among the different age groups.

Four to seven muscles were used for each drug in each group. Cumulative dose-response relationships (1 to $300 \mu \mathrm{M})$ were obtained using a single drug for each muscle. Stock solutions prepared in DMSO were diluted with Krebs bicarbonate buffer, resulting in a final DMSO concentration of less than $1 \%$. DMSO at this concentration had no significant effect on contractility in control studies in all age groups. Maximal drug effects occurred between 5 and $10 \mathrm{~min}$ (independent of age group) and were recorded approximately $10 \mathrm{~min}$ after the addition of each drug dose. Thus, cumulative dose-response studies required no longer than 90 min to complete.

Inhibition of $P D E$ activities. Ventricular myocardium was trimmed of atria, great vessels, and gross epicardial fat. All procedures were performed at $4^{\circ} \mathrm{C}$. Approximately $15-20 \mathrm{~g}$ of tissue were used for each adult preparation (two adult hearts were pooled for each different preparation), and 7-10 g were used for immature and newborn preparations (this required pooling of several littermates). The myocardium was minced and added to 10 volumes $(1 \mathrm{~g} / 10 \mathrm{~mL})$ of buffer containing 4 $\mathrm{mM}$ EDTA, $20 \mathrm{mM} \mathrm{NaCl}, 10 \mathrm{mM}$ benzamidine, $1 \mathrm{KIU} / \mathrm{mL}$ aprotinin, $2 \mu \mathrm{M}$ leupeptin, $10 \mu \mathrm{M} n$-tosyl-1-lysine chloromethyl ketone, and $2 \mu \mathrm{M}$ pepstatin-A. Samples were homogenized with a Brinkmann Polytron PT-20 (Brinkmann Instruments, Co., Westbury, NY) using three 30 -s bursts at half-maximal setting. Homogenates of AD myocardium were filtered through gauze to remove fat, but this step was not required for the younger age groups. Homogenates were centrifuged for $20 \mathrm{~min}$ at $30000 \times$ $g$. For NB and IM preparations, the resultant supernatants were suspended in ethylene glycol (final concentration of ethylene glycol was $30 \%$ ) and stored briefly at $-20^{\circ} \mathrm{C}$ until analyzed. For adult preparations, the high-affinity cAMP activities in the supernatant were partially purified (peak III) by anion exchange chromatography as previously described from our laboratory $(8$, 12). Adult samples were suspended in $30 \%$ ethylene glycol and stored briefly at $-20^{\circ} \mathrm{C}$ for subsequent analyses. These methods for obtaining cytosolic preparations result in recovery of approximately $85-90 \%$ of total homogenate high-affinity cyclic nucleotide PDE activity regardless of age group.

PDE assays were performed according to routine procedures in our laboratory $(8,12,16,23,24)$. Cytosolic fractions were assayed for hydrolysis of either $0.25 \mu \mathrm{M}\left[{ }^{3} \mathrm{H}\right] \mathrm{cAMP}$ or $1 \mu \mathrm{M}\left[{ }^{3} \mathrm{H}\right]$ cGMP. Under these conditions with low substrate concentrations, the predominant PDE activities detected are those with high substrate affinity. The $\mathrm{IC}_{50}$ determinations were performed using 10 different inhibitor concentrations ranging from 0.01 to $300 \mu \mathrm{M}$. Drugs were dissolved in $100 \%$ DMSO and then diluted appropriately. Control experiments using the same concentrations of DMSO, but without drug, demonstrated that the highest concentration of DMSO inhibited basal activity by approximately $20 \%$ in each age group. However, the effects of DMSO at concentrations near drug $\mathrm{IC}_{50}$ values were negligible (basal activity inhibited by less than $5 \%$ ).

Protein was determined by the Bradford method (25), using BSA as the standard.

Drugs. RO 20-1724 [4-(3-butoxy-4-methoxybenzyl)-2-imidazolidinone] was a gift from Dr. Herbert Sheppard of HoffmanLaRoche, Inc. (Nutley, NJ), SQ 65,442 [1-ethyl-4-(ethylthio)$1 \mathrm{H}$-pyrazolo[3,4-b]-pyridine-5-carboxylic acid, ethyl ester] was donated by Dr. Jon Norman of E. R. Squibb and Sons, Inc. (New Brunswick, NJ), and trequinsin [9,10-dimethoxy-2-mesitylimino-3-methyl-3,4,6,7-tetrahydro-2H-pyrimido $(6,1-\mathrm{A})$ isoquinoline-4-one-hydrochloride] was from Hoechst-Roussel Pharmaceuticals, Inc. (Somerville, NJ).

Statistical analyses. Contractile data were analyzed using oneway analysis of variance. If the $F$ ratio indicated a significant difference $(p<0.05)$, a protected Fisher's Least Significant Difference test was used to provide intergroup comparisons. Results are expressed as mean \pm SEM.

The individual drug concentrations required to inhibit basal PDE activity by $50 \%$ were defined as the $\mathrm{IC}_{50}$ values. $\mathrm{IC}_{50}$ values and the error estimate of these values based upon $95 \%$ confidence limits of the full dose-response curves (10 different concentrations of inhibitor) were determined using the IBM PC version of Curve Fit (M. L. Jaffe Associates, Silver Spring, MD). This program calculates parameters for a four-parameter logistic function:

$$
Y=\frac{a-d}{1+(X / c)^{b}}+d
$$

where $\mathrm{X}=$ dose, $\mathrm{Y}=\%$ inhibition, $\mathrm{a}=\mathrm{Y}$ at zero dose, $\mathrm{d}=\mathrm{Y}$ at infinite dose, $\mathrm{c}=\mathrm{Y}$ halfway between $\mathrm{a}$ and $\mathrm{d}\left(\mathrm{IC}_{50}\right)$, and $\mathrm{b}=$ "slope factor." The computation uses the $2+2$ linear regression approach described by Rodbard and Hutt (26). 


\section{RESULTS}

Contractile responses. Table 1 contains the pretreatment control values for each index of contractile function measured in all three age groups. Inotropic effects were assessed by comparing drug-induced changes in the $\mathrm{dT} / \mathrm{dt}$. Because of inherent agedependent differences in basal contractile function, drug responses are expressed as a percentage of the pretreatment control values.

Figure 1 illustrates the inotropic responses in papillary muscles from each age group exposed to increasing concentrations of $\mathrm{RO}$ 20-1724. In the NB group, $100 \mu \mathrm{M}$ RO 20-1724 reduced dT/dt to $67 \pm 4 \%$ of the pretreatment control value. However, in papillary muscles from IM and AD rabbits, $100 \mu \mathrm{M}$ RO 20-1724 produced a slight increase in $\mathrm{dT} / \mathrm{dt}$ to $127 \pm 2$ and $115 \pm 3 \%$ of control, respectively. As demonstrated in Table 2, there were age-related differences in the effects of RO 20-1724 on other indices of contractile function. In the NB group, RO 20-1724 exerted a negative lusitropic effect (prolonged $90 \%$ relaxation time and reduced $-\mathrm{dT} / \mathrm{dt}$ ) and elevated RT. Opposite responses were observed in the IM and AD groups with regard to these indices of contractile function.

SQ 65,442 , another selective inhibitor of cAMP specific class IV PDE, produced a dose-dependent decrease in $\mathrm{dT} / \mathrm{dt}$ in all age groups (Fig. 2). At $100 \mu \mathrm{M}$ SQ 65,442, the reduction in $\mathrm{dT} / \mathrm{dt}$ was not significantly different among the three different groups (65-75\% of control). In addition to a negative inotropic effect, SQ 65,442 increased RT, prolonged the duration of contraction, and impaired relaxation in each age group (Table 2).

The effects of trequinsin on $\mathrm{dT} / \mathrm{dt}$ in each age group are illustrated in Figure 3. The maximal positive inotropic response in the NB group was significantly greater $(p<0.01)$ than that observed in either the IM or AD group. The improvement in contractility in the NB group was accompanied by a positive lusitropic effect (increased $-\mathrm{dT} / \mathrm{dt}$; Table 2) that was less pronounced in the other two age groups $(p<0.05)$.

To facilitate comparisons of the maximal inotropic effect for such age group and each drug, results for dT/dt at $100 \mu \mathrm{M}$ drug concentration are summarized in Figure 4. The inotropic effects of the three PDE inhibitors differed among the age groups. By 2 wk of age (IM group) the AD pattern of responses was fully developed. Papillary muscles from NB animals exhibited a fall in dT/dt in response to RO 20-1724 and SQ 65,442. In contrast, an NB-specific positive inotropic effect occurred after exposure to trequinsin.

Inhibition of PDE activities. The ability of the drugs to inhibit cytosolic cAMP hydrolysis was determined over a range of drug concentrations to provide an index of the sensitivity to inhibition ( $\mathrm{IC}_{50}$ values; Table 3 ). $\mathrm{IC}_{50}$ values were obtained using $0.25 \mu \mathrm{M}$ CAMP as substrate to evaluate drug effects on high-affinity cAMP PDE activity in the cytosolic mixture. As shown in Table 3, all three drugs are potent inhibitors of cytosolic cAMP hydrolysis, with NB preparations exhibiting slightly greater sensitivity. However, within the $\mathrm{NB}$ group, $\mathrm{IC}_{50}$ values for each drug were comparable despite marked differences in the contractile responses to these agents. Because differences in sensitivity of cAMP hydrolysis did not adequately account for differences in inotropic responsiveness and because cGMP may be an important modulator of cAMP PDE activities, the ability of trequinsin and SQ 65,442 to inhibit cGMP hydrolysis was determined in each age group (Table 4). Trequinsin was found to be a more potent inhibitor of cGMP hydrolysis than SQ 65,442 , but no consistent pattern of age-related effects was apparent.

\section{DISCUSSION}

Previous developmental studies have demonstrated that NB animals are relatively insensitive to the positive inotropic effects of milrinone, a selective inhibitor of cGMP-inhibited class III PDE in the SR (14-17). We attributed this lack of response in $\mathrm{NB}$ rabbits to a relative deficiency of SR-associated class III PDE activity (12). Based upon the observation that cytosolic highaffinity cAMP activity is predominantly the cAMP-specific class IV form in the NB rabbit heart (12), we postulated that selective inhibitors of this activity would exert a positive inotropic effect in NB myocardium. However, results from the present study indicate that two different selective inhibitors of cAMP-specific class IV PDE (SQ 65,442 and RO 20-1724) did not increase myocardial contractility in papillary muscles isolated from NB rabbits. This inotropic unresponsiveness occurred despite equivalent or even slightly greater sensitivity to inhibition of cAMP hydrolysis. The increased sensitivity to inhibition of cAMP hydrolysis by these drugs in the NB group is consistent with recognized age-related differences in the relative proportion of the two predominant forms of high-affinity cAMP PDE in the cytosol.

As anticipated, these agents had little or no positive inotropic effect in AD papillary muscles under these conditions (27). Contractile responses and drug effects on PDE activities were comparable in the IM and $\mathrm{AD}$ groups. These results along with previous observations (23), suggest that by $14 \mathrm{~d}$ of age, the cyclic nucleotide PDE system in the rabbit has undergone substantial development. Future studies of this system might benefit from incorporating a late fetal and/or 7-d-old age group.

The different pattern of responses to RO 20-1724 and SQ 65,442 in mature myocardium suggests that subtle differences in PDE inhibition profiles may produce different contractile responses. In addition, these drugs may exert unrecognized effects on other subcellular mechanisms involved in the regulation of myocardial contractility. Conceivably, the negative inotropic effect of SQ 65,442 could be due to an additional drug action that is yet to be defined.

The lack of a positive inotropic response to high-affinity cAMP PDE inhibitors in NB myocardium is not attributable to an inability to respond to increased intracellular levels of cAMP, inasmuch as isoproterenol or forskolin produce a significant positive inotropic effect in NB rabbits (16). The relatively greater magnitude of the forskolin response observed in NB suggests that the mechanisms for cAMP hydrolysis are incompletely developed at birth in the rabbit. Trequinsin, a potent but much less selective PDE inhibitor, was used to test whether or not generalized PDE inhibition would improve contractility in the NB group. Unexpectedly, trequinsin exerted an age-specific positive inotropic effect. Papillary muscles from NB animals exhibited a 3.5 -fold increase in $\mathrm{dT} / \mathrm{dt}$. In contrast, there was only a very modest effect of trequinsin in the IM and $\mathrm{AD}$ groups.

At present, the specific PDE isozyme(s) responsible for the response to trequinsin in NB rabbits is unclear. Recently, Muller et al. (27) have shown that cAMP-specific class IV PDE may assume a role in regulating cardiac contractility in mature guinea

Table 1. Pretreatment contractile function in NB, IM, and AD rabbit papillary muscles*

\begin{tabular}{ccccccc}
\hline Age group & $\begin{array}{c}\mathrm{dT} / \mathrm{dt} \\
\left(\mathrm{g} / \mathrm{s} / \mathrm{mm}^{2}\right)\end{array}$ & $\begin{array}{c}\mathrm{DT} \\
\left(\mathrm{g} / \mathrm{mm}^{2}\right)\end{array}$ & $\begin{array}{c}\mathrm{RT} \\
\left(\mathrm{g} / \mathrm{mm}^{2}\right)\end{array}$ & $\begin{array}{c}\text { TPT } \\
(\mathrm{ms})\end{array}$ & $\begin{array}{c}90 \% \mathrm{RelT} \\
(\mathrm{ms})\end{array}$ & $\begin{array}{c}-\mathrm{dT} / \mathrm{dt} \\
\left(\mathrm{g} / \mathrm{s} / \mathrm{mm}^{2}\right)\end{array}$ \\
\hline NB & $4.23 \pm 0.21$ & $0.72 \pm 0.04$ & $0.70 \pm 0.08$ & $197 \pm 4$ & $183 \pm 4$ & $4.89 \pm 0.38$ \\
IM & $11.58 \pm 0.64$ & $1.62 \pm 0.06$ & $0.32 \pm 0.03$ & $185 \pm 4$ & $209 \pm 10$ & $13.39 \pm 0.30$ \\
AD & $15.47 \pm 0.70$ & $2.30 \pm 0.15$ & $0.48 \pm 0.02$ & $195 \pm 3$ & $153 \pm 4$ & $20.79 \pm 1.27$ \\
\hline
\end{tabular}

${ }^{*}$ Results are presented as mean \pm SEM; $n=14-17$ in each group. DT, developed tension; TPT, time to peak tension; and $90 \%$ RelT, time to $90 \%$ relaxation. 


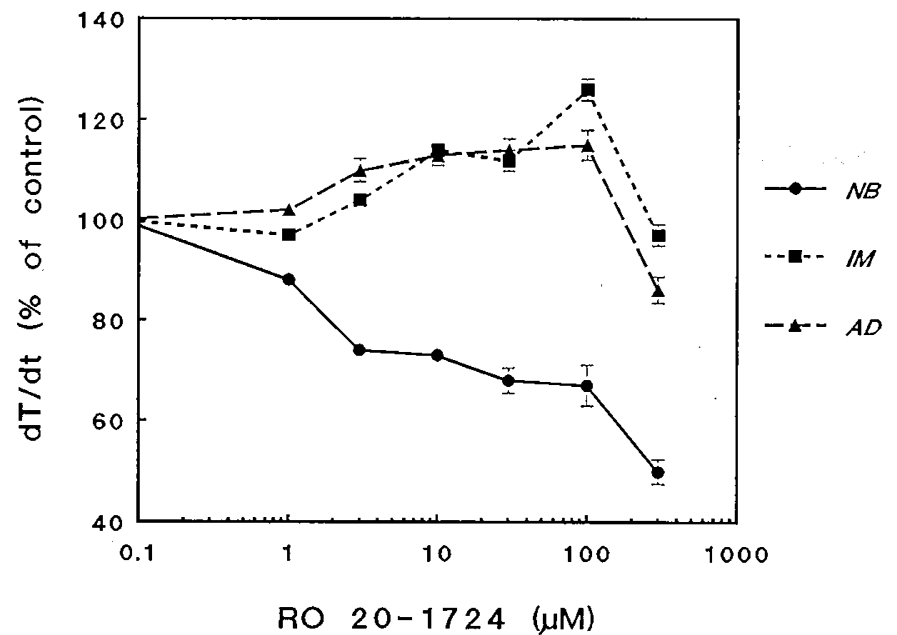

Fig. 1. Effect of RO 20-1724 on dT/dt in right ventricular papillary muscles isolated from NB, IM, and AD rabbits. Results are normalized to $\%$ of pretreatment control values. Each point represents the mean ( $n$ $=4-7)$, and the bars indicate SEM.

Table 2. Drug effects on contractile function*

\begin{tabular}{lrrrrr}
\hline & \multicolumn{1}{c}{ RT } & DT & TPT & $90 \%$ RelT & -dT/dt \\
\hline RO 20-1724 & & & & & \\
NB & $123 \pm 5$ & $79 \pm 1$ & $105 \pm 2$ & $112 \pm 1$ & $73 \pm 2$ \\
IM & $80 \pm 2$ & $105 \pm 2$ & $88 \pm 4$ & $93 \pm 2$ & $107 \pm 4$ \\
AD & $86 \pm 1$ & $100 \pm 2$ & $84 \pm 1$ & $86 \pm 2$ & $113 \pm 2$ \\
SQ 65,442 & & & & & \\
NB & $134 \pm 6$ & $80 \pm 2$ & $121 \pm 1$ & $115 \pm 2$ & $56 \pm 1$ \\
IM & $103 \pm 1$ & $83 \pm 1$ & $113 \pm 1$ & $121 \pm 1$ & $83 \pm 2$ \\
AD & $107 \pm 1$ & $85 \pm 1$ & $118 \pm 1$ & $126 \pm 3$ & $74 \pm 4$ \\
Trequinsin & & & & & \\
NB & $83 \pm 3$ & $244 \pm 12$ & $73 \pm 1$ & $77 \pm 2$ & $325 \pm 18$ \\
IM & $87 \pm 2$ & $134 \pm 5$ & $81 \pm 1$ & $71 \pm 1$ & $144 \pm 3$ \\
AD & $87 \pm 1$ & $106 \pm 2$ & $88 \pm 1$ & $94 \pm 1$ & $127 \pm 5$ \\
\hline
\end{tabular}

* Results are expressed as \% of pretreatment control values (mean \pm SEM); $n=4-7$ in each group. Data presented were obtained at $100 \mu \mathrm{M}$ drug concentration. DT, developed tension; TPT, time to peak tension; and $90 \%$ RelT, time to $90 \%$ relaxation.

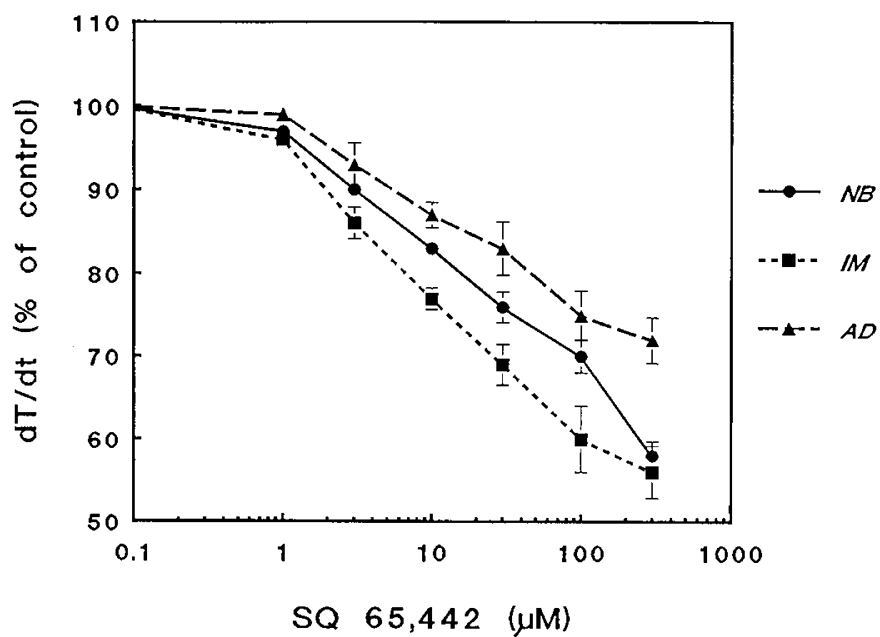

Fig. 2. Effect of SQ 65,442 on $\mathrm{dT} / \mathrm{dt}$ in right ventricular papillary muscles isolated from NB, IM, and AD rabbits. Results are normalized to $\%$ of pretreatment control values. Each point represents the mean ( $n$ $=4-7)$, and the bars indicate SEM.

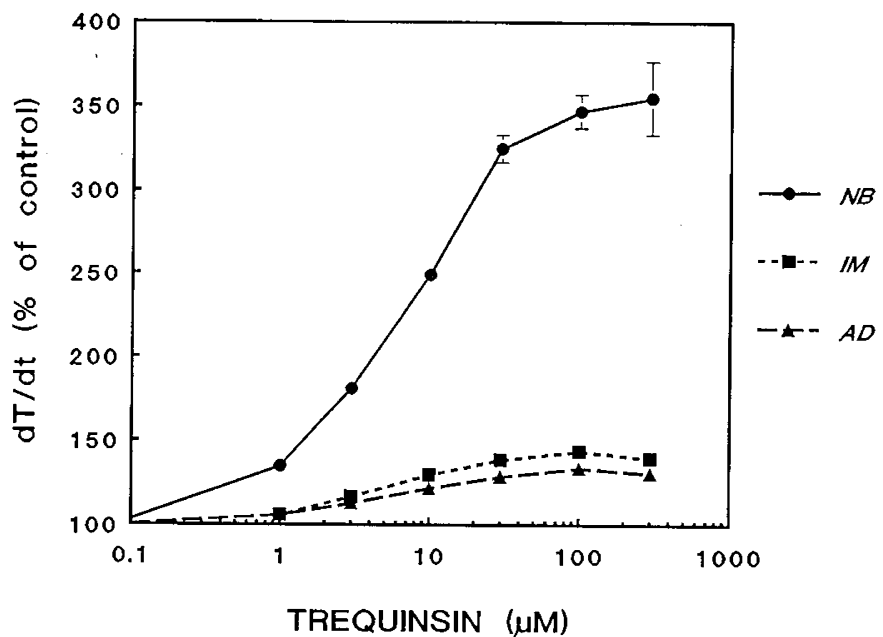

Fig. 3. Effect of trequinsin on $\mathrm{dT} / \mathrm{dt}$ in right ventricular papillary muscles isolated from NB, IM, and AD rabbits. Results are normalized to $\%$ of pretreatment control values. Each point represents the mean ( $n$ $=4-7)$, and the bars indicate SEM.

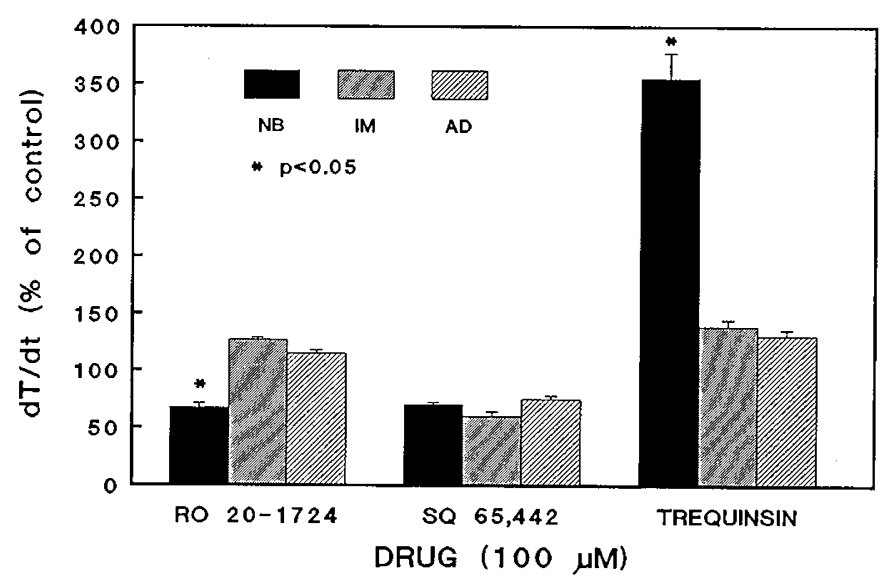

Fig. 4. Comparisons of inotropic effects at $100 \mu \mathrm{M}$ drug concentration. *, NB response differs from AD and IM.

Table 3. Inhibition of cytosolic cAMP hydrolysis*

\begin{tabular}{lccc}
\hline & \multicolumn{3}{c}{$\mathrm{IC}_{50}$ value $(\mu \mathrm{M})$} \\
\cline { 2 - 4 } Drug & $\mathrm{NB}$ & $\mathrm{IM}$ & $\mathrm{AD}$ \\
\hline Trequinsin & $0.3 \pm 0.1$ & $0.7 \pm 0.1$ & $1.0 \pm 0.1$ \\
SQ 65,442 & $0.4 \pm 0.1$ & $1.7 \pm 0.2$ & $0.2 \pm 0.1$ \\
RO 20-1724 & $0.9 \pm 0.1$ & $1.9 \pm 0.3$ & $0.5 \pm 0.2$ \\
\hline
\end{tabular}

${ }^{*}$ Results represent mean \pm SEM computed from the combined doseresponse curves. PDE assays were performed using $0.25 \mu \mathrm{M}$ cAMP as substrate.

Table 4. Inhibition of cytosolic cGMP hydrolysis*

\begin{tabular}{crrr}
\hline & \multicolumn{3}{c}{$\mathrm{IC}_{50}$ value $(\mu \mathrm{M})$} \\
\cline { 2 - 4 } Drug & \multicolumn{1}{c}{$\mathrm{NB}$} & \multicolumn{1}{c}{$\mathrm{IM}$} & \multicolumn{1}{c}{$\mathrm{AD}$} \\
\hline Trequinsin & $2.5 \pm 0.3$ & $1.9 \pm 0.3$ & $4.2 \pm 0.4$ \\
SQ 65,442 & $26.8 \pm 3.7$ & $56.6 \pm 7.2$ & $37.4 \pm 1.8$ \\
\hline
\end{tabular}

* Results represent mean \pm SEM computed from the combined doseresponse curves. PDE assays were performed using $1.0 \mu \mathrm{M}$ cGMP as substrate.

pig atria under conditions in which adenylate cyclase activity is stimulated. However, data from the present study using the selective inhibitors RO 20-1724 and SQ 65,442 suggest that the cAMP-specific class IV PDE does not contribute to the inotropic response elicited by trequinsin in the NB rabbit. Sensitivity to 
inhibition of high-affinity cAMP PDE, as indexed by $\mathrm{IC}_{50}$ values (Table 3), was comparable for each of the three drugs in the NB group, yet only trequinsin increased $\mathrm{dT} / \mathrm{dt}$. Furthermore, the previously reported lack of a positive inotropic response to selective inhibitors (e.g. milrinone) of the SR-associated cGMPinhibited class III cAMP PDE (14-17) suggests that inhibition of this PDE isoform alone is not sufficient to account for the mechanism of action of trequinsin in the NB.

Trequinsin inhibited cGMP hydrolysis in the low micromolar range in all three age groups, consistent with the more generalized PDE inhibitory effects of this drug (in contrast to SQ 65,442 $\mathrm{IC}_{50}$ values for cGMP hydrolysis). However, inhibition of cGMP hydrolysis alone does not appear to account for the age-related differences in inotropic responsiveness, because the trequinsin $\mathrm{IC}_{50}$ values were quite comparable in the three age groups.

Taken in concert, the current results and the aforementioned previous findings provide evidence to support the concept that contractility and drug responsiveness in NB rabbit myocardium are modulated by PDE isoforms that differ from the PDE that predominant in the mature heart. It may be that a calciumcalmodulin-stimulated PDE (class I) or a cGMP-stimulated PDE (class II) assumes a relatively greater role in regulating cAMP metabolism in the developing heart. Alternatively, the interaction of multiple PDE isoforms may be a more important determinant of the overall inotropic response, particularly in the developing heart. In addition, it is conceivable that trequinsin may exert unrecognized effects other than PDE inhibition that assume an important role in mediating the response in the NB heart.

Drug effects in homogenates/cell-free systems may not always correlate with the pharmacodynamic response in intact tissues. For example, the ability of cardiotonic drugs to inhibit the SRassociated, cGMP-inhibited, high-affinity cAMP PDE correlates well with contractile responses in $\mathrm{AD}$ rabbit myocardium. In contrast, inhibition of cAMP hydrolysis by various drugs in rat brain homogenates does not correlate with the ability of these drugs to inhibit cAMP turnover in intact brain tissue slices (19). Based upon our current results, we speculate that trequinsin would increase cAMP levels and activate cAMP-dependent protein kinase in NB myocardium but not in preparations from mature rabbits. Additional studies using inhibitors of class I and class II PDE could be tested to probe the role of these other PDE activities in regulating contractile function in the developing heart.

In summary, we have shown that selective inhibitors of cAMPspecific class IV PDE do not increase contractility in NB rabbit myocardium. The age-specific positive inotropic effect of trequinsin suggests that the regulation of contractile function during perinatal maturation involves PDE isoforms that differ from those exerting a major influence in the mature heart. Furthermore, it may be possible to exploit these age-dependent differences by formulating inotropic agents (such as trequinsin) that exhibit selective therapeutic efficacy in the developing heart.

\section{REFERENCES}

1. Tada M, Katz AM 1982 Phosphorylation of the sarcoplasmic reticulum and sarcolemma. Annu Rey Physiol 44:401-423

2. Ahn HS, Eardley D, Watkins R, Prioli N 1986 Effects of several newer cardiotonic drugs on cardiac cyclic AMP metabolism. Biochem Pharmacol 35:1112-1121

3. Olson EM, Kim D, Smith TW, Marsh JD 1987 Mechanism of the positive inotropic effect of milrinone in cultured embryonic chick ventricular cells. $J$ Mol Cell Cardiol 19:95-104

4. Hartzell HC 1988 Regulation of cardiac ion channels by catecholamines, acetylcholine and second messenger systems. Prog Biophys Mol Biol 52: :165-247

5. Muller B, Lugnier C, Stoclet JC 1990 Implication of cyclic AMP in the positive inotropic effects of cyclic GMP-inhibited cyclic AMP phosphodiesterase inhibitors on guinea pig isolated left atria. J Cardiovasc Pharmacol 15: $444-451$

6. Beavo J 1990 Multiple phosphodiesterase: isozymes, background, nomenclature and implications. In: Beavo J, Houslay MD (eds) Cyclic Nucleotide Phosphodiesterases: Structure, Regulation and Drug Action. John Wiley \& Sons, West Sussex, UK, pp 3-15

7. Weishaar RE, Kobylarz-Singer DC, Steffen RP, Kaplan HR 1987 Subclasses of cyclic AMP-specific phosphodiesterase in left ventricular muscle and their involvement in regulating myocardial contractility. Circ Res 61:539-547

8. Kithas PA, Artman M, Thompson WJ, Strada SJ 1988 Subcellular distribution of high-affinity type IV cyclic nucleotide phosphodiesterase activity in rabbit ventricular myocardium: relations to the effects of cardiotonic drugs. Circ Res 62:782-789

9. Harrison SA, Reifsnyder DH, Gallis B, Cadd GG, Beavo JA 1986 Isolation and characterization of bovine cardiac muscle cGMP-inhibited phosphodiesterase: a receptor for new cardiotonic drugs. Mol Pharmacol 29:506-514

10. Kauffman RA, Utterback BG, Robertson DW 1989 Characterization and pharmacological relevance of high affinity binding sites for $\left[{ }^{3} \mathrm{H}\right] \mathrm{LY} 186126$, a cardiotonic phosphodiesterase inhibitor, in canine cardiac membranes. Circ Res 65:154-163

11. Artman M, Robertson DW, Mahony L, Thompson WJ 1989 Analysis of the binding sites for the cardiotonic phosphodiesterase inhibitor $\left[{ }^{3} \mathrm{H}\right] \mathrm{LY} 186126$ in ventricular myocardium. Mol Pharmacol 36:302-311

12. Kithas PA, Artman M, Thompson WJ, Strada SJ 1989 Subcellular distribution of high affinity type IV cyclic AMP phosphodiesterase activities in rabbit ventricular myocardium: relations to post-natal maturation. $\mathbf{J}$ Mol Cel Cardiol 21:507-517

13. Silver PJ, Harris AL, Canniff PC, Lepore RE, Bentley RG, Hamel LT, Evans DB 1989 Phosphodiesterase isozyme inhibition, activation of the cAMP system, and positive inotropy mediated by milrinone in isolated guinea pig cardiac muscle. J Cardiovasc Pharmacol 13:530-540

14. Binah O, Sodowick B, Vulliemoz Y, Danilo Jr P, Rosen M 1986 The inotropic effects of amrinone and milrinone on neonatal and young canine cardiac muscle. Circulation 73(suppl III):III-46-III-51

15. Ogawa S-I, Nakanishi T, Kamata K, Takao A 1987 Effect of milrinone on myocardial mechanical function and cyclic AMP content in the fetal rabbit. Pediatr Res 22:282-285

16. Artman M, Kithas PA, Wike JS, Strada SJ 1988 Inotropic responses change during postnatal maturation in rabbit. Am J Physiol 255:H335-H342

17. Klitzner TS, Shapir Y, Ravin R, Friedman WF 1990 The biphasic effect of amrinone on tension development in newborn mammalian myocardium. Pediatr Res 27:144-147

18. Weishaar RE, Kobylarz-Singer DC, Kaplan HR 1987 Subclasses of cyclic AMP phosphodiesterase in cardiac muscle. J Mol Cell Cardiol 19:1025-1036

19. Whalin ME, Garrett RL, Thompson WJ, Strada SJ 1989 Correlation of cellfree brain cyclic nucleotide phosphodiesterase activities to cyclic AMP decay in intact brain slices. Sec Mess Phosphoprot 12:311-325

20. Epstein PM, Strada SJ, Sarada K, Thompson WJ 1982 Catalytic and kinetic properties of purified high-affinity cyclic AMP phosphodiesterase from dog kidney. Arch Biochem Biophys 218:119-133

21. Lugnier C, Schoeffler P, Le Bec A, Strouthou E, Stoclet JC 1986 Selective inhibition of cyclic nucleotide phosphodiesterases of human, bovine and rat aorta. Biochem Pharmacol 35:1743-1751

22. Souness JE, Diocee BK, Martin W, Moodie SA 1990 Pig aortic endothelialcell cyclic nucleotide phosphodiesterases: use of phosphodiesterase inhibitors to evaluate their roles in regulating cyclic nucleotide levels in intact cells. Biochem J 266:127-132

23. Artman M, Kithas PA, Wike JS, Crump DB, Strada SJ 1988 Inotropic responses to cyclic nucleotide phosphodiesterase inhibitors in immature and adult rabbit myocardium. J Cardiovasc Pharmacol 13:146-154

24. Thompson WJ, Strada SJ 1984 Cyclic nucleotide phosphodiesterase (PDE). In: Bergmeyer HU (ed) Methods of Enzymatic Analysis. Verlag Chemie, Weinheim, pp 127-134

25. Bradford MM 1976 A rapid and sensitive method for quantitation of microgram quantities of protein utilizing the principle of protein-dye binding. Anal Biochem 72:248-254

26. Rodbard D, Hutt DM 1974 Statistical analysis of radioimmunoassay and immunoradiometric (labeled antibody) assays: a generalized weighted, iterative least-squares method for logistic curve fitting. In: Proceedings, Symposium on Radioimmunoassay and Related Procedures in Medicine, International Atomic Energy Agency. Unipub, New York, pp 165-192

27. Muller B, Lugnier C, Stoclet JC 1990 Involvement of rolipram-sensitive cyclic AMP phosphodiesterase in the regulation of cardiac contraction. J Cardiovasc Pharmacol 16:796-803 